\title{
Risk of hypoglycaemia with insulin degludec versus insulin glargine U300 in insulin-treated patients with type 2 diabetes: the randomised, head-to-head CONCLUDE trial
}

\author{
Athena Philis-Tsimikas ${ }^{1}$ (D) David C. Klonoff ${ }^{2} \cdot$ Kamlesh Khunti $^{3} \cdot$ Harpreet S. Bajaj $^{4} \cdot$ Lawrence A. Leiter $^{5}$. \\ Melissa V. Hansen ${ }^{6}$. Lone N. Troelsen ${ }^{6}$. Steen Ladelund ${ }^{6}$. Simon Heller ${ }^{7}$. Thomas R. Pieber ${ }^{8}$. on behalf of the \\ CONCLUDE Study Group
}

Received: 9 September 2019 / Accepted: 4 December 2019/Published online: 27 January 2020

(C) The Author(s) 2020

\begin{abstract}
Aims/hypothesis A head-to-head randomised trial was conducted to evaluate hypoglycaemia safety with insulin degludec $200 \mathrm{U} /$ $\mathrm{ml}$ (degludec U200) and insulin glargine $300 \mathrm{U} / \mathrm{ml}$ (glargine U300) in individuals with type 2 diabetes treated with basal insulin. Methods This randomised (1:1), open-label, treat-to-target, multinational trial included individuals with type 2 diabetes, aged $\geq 18$ years with $\mathrm{HbA}_{1 \mathrm{c}} \leq 80 \mathrm{mmol} / \mathrm{mol}(9.5 \%)$ and $\mathrm{BMI} \leq 45 \mathrm{~kg} / \mathrm{m}^{2}$. Participants were previously treated with basal insulin with or without oral glucose-lowering drugs (excluding insulin secretagogues) and had to fulfil at least one predefined criterion for hypoglycaemia risk. Both degludec U200 and glargine U300 were similarly titrated to a fasting blood glucose target of 4.0-5.0 mmol/1. Endpoints were assessed during a 36 week maintenance period and a total treatment period up to 88 weeks. There were three hypoglycaemia endpoints: (1) overall symptomatic hypoglycaemia (either severe, an event requiring third-party assistance, or confirmed by blood glucose [<3.1 mmol/1] with symptoms); (2) nocturnal symptomatic hypoglycaemia (severe or confirmed by blood glucose with symptoms, between 00:01 and 05:59 h); and (3) severe hypoglycaemia. The primary endpoint was the number of overall symptomatic hypoglycaemic events in the maintenance period. Secondary hypoglycaemia endpoints included the number of nocturnal symptomatic events and number of severe hypoglycaemic events during the maintenance period.

Results Of the 1609 randomised participants, 733 of $805(91.1 \%)$ in the degludec U200 arm and 734 of 804 (91.3\%) in the glargine U300 arm completed the trial ( $87.3 \%$ and $87.8 \%$ completed on treatment, respectively). Baseline characteristics were comparable between the two treatment arms. For the primary endpoint, the rate of overall symptomatic hypoglycaemia was not significantly lower with degludec U200 vs glargine U300 (rate ratio [RR] 0.88 [95\% CI 0.73, 1.06]). As there was no significant difference between treatments for the primary endpoint, the confirmatory testing procedure for superiority was stopped. The prespecified confirmatory secondary hypoglycaemia endpoints were analysed using pre-specified statistical models but were now considered exploratory. These endpoints showed a lower rate of nocturnal symptomatic hypoglycaemia (RR 0.63 [95\% CI 0.48 , 0.84]) and severe hypoglycaemia (RR 0.20 [95\% CI 0.07, 0.57]) with degludec U200 vs glargine U300.
\end{abstract}

Electronic supplementary material The online version of this article (https://doi.org/10.1007/s00125-019-05080-9) contains peer-reviewed but unedited supplementary material, which is available to authorised users.

Athena Philis-Tsimikas

Philis-Tsimikas.Athena@scrippshealth.org

1 Scripps Whittier Diabetes Institute, 10140 Campus Point Drive, Suite 200, San Diego, CA 92121, USA

2 Diabetes Research Institute, Mills-Peninsula Medical Center, San Mateo, CA, USA

3 Diabetes Research Centre, University of Leicester, Leicester, UK

4 LMC Diabetes and Endocrinology, Brampton, ON, Canada
5 Li Ka Shing Knowledge Institute, Division of Endocrinology \& Metabolism, St Michael's Hospital, University of Toronto, Toronto, ON, Canada

6 Novo Nordisk A/S, Søborg, Denmark

7 Academic Unit of Diabetes, Endocrinology and Metabolism, University of Sheffield, Sheffield, UK

8 Division of Endocrinology and Diabetology, Department of Internal Medicine, Medical University of Graz, Graz, Austria 


\section{Research in context}

\section{What is already known about this subject?}

- Minimising hypoglycaemia is important for people with type 2 diabetes using insulin

- Use of second-generation long-acting basal insulins, insulin degludec $200 \mathrm{U} / \mathrm{ml}$ (degludec U200) and insulin glargine $300 \mathrm{U} / \mathrm{ml}$ (glargine U300), compared with insulin glargine $100 \mathrm{U} / \mathrm{ml}$ (glargine U100), has been shown to result in a lower risk of hypoglycaemia

\section{What is the key question?}

- Is there a difference in the risk of hypoglycaemia with degludec U200 compared with glargine U300 in insulintreated patients with type 2 diabetes when similarly titrated to a target fasting blood glucose of $4.0-5.0 \mathrm{mmol} / \mathrm{I}$ ?

\section{What are the new findings?}

- There was no significant difference in the rate of overall symptomatic hypoglycaemia with degludec U200 vs glargine U300 in the maintenance period in insulin-treated individuals

- The rates of nocturnal symptomatic and severe hypoglycaemia were nominally significantly lower with degludec U200 compared with glargine U300 during the maintenance period

\section{How might this impact on clinical practice in the foreseeable future?}

- Results from the CONCLUDE trial add to the published literature on the latest basal insulins, degludec U200 and glargine U300, informing healthcare providers and health systems on how to achieve blood glucose targets for their patients more safely

Conclusions/interpretation There was no significant difference in the rate of overall symptomatic hypoglycaemia with degludec U200 vs glargine U300 in the maintenance period. The rates of nocturnal symptomatic and severe hypoglycaemia were nominally significantly lower with degludec U200 during the maintenance period compared with glargine U300.

Trial registration ClinicalTrials.gov NCT03078478

Funding This trial was funded by Novo Nordisk (Bagsvaerd, Denmark)

Keywords Clinical science · Hypoglycaemia · Insulin degludec $\cdot$ Insulin glargine $\cdot$ Insulin therapy

$\begin{array}{ll}\text { Abbreviations } \\ \text { Degludec U200 } \\ \text { FPG } & \text { Insulin degludec } 200 \mathrm{U} / \mathrm{ml} \\ \text { Glargine U100 } & \text { Fasting plasma glucose } \\ \text { Glargine U300 } & \text { Insulin glargine } 100 \mathrm{U} / \mathrm{ml} \\ \text { MMRM } & \text { Mixed model of repeated measures } \\ \text { OAD } & \text { Oral glucose-lowering drug } \\ \text { PYE } & \text { Person-years of exposure } \\ \text { RR } & \text { Rate ratio } \\ \text { SMBG } & \text { Self-measured blood glucose }\end{array}$

\section{Introduction}

Hypoglycaemia is a known complication of insulin treatment and is acknowledged as the main limiting factor for achieving tight glycaemic control $[1,2]$. The two most recently developed second-generation, longer-acting basal insulins, insulin degludec and insulin glargine $300 \mathrm{U} / \mathrm{ml}$ (glargine U300), have flatter and more stable steady-state pharmacokinetic and pharmacodynamic profiles compared with long-acting insulin glargine $100 \mathrm{U} / \mathrm{ml}$ (glargine U100) [3-6]. Insulin degludec has a lower day-to-day variability in glucose-lowering effect compared with glargine U100 and glargine U300 [6, 7], whereas there are contradictory reports regarding within-day variability when comparing insulin degludec and glargine U300 [7, 8].

Insulin degludec and glargine U300 have been shown to be associated with a lower risk of hypoglycaemia, at equivalent glycaemic control compared with glargine U100 in individuals with type 2 diabetes [9-20]. Glargine U300 is a concentrated formulation of glargine U100 and has also been shown to be as effective as glargine U100 in terms of glycaemic control in individuals with type 2 diabetes, but with a higher (12-14\%) basal insulin dose requirement [14-20]. Recent results in insulin-naive individuals with type 2 diabetes revealed similar $\mathrm{HbA}_{1 \mathrm{c}}$ reductions for insulin degludec and glargine U300 [21]. This trial also reported a similar overall 
risk of hypoglycaemia between the two insulins and a lower rate of hypoglycaemia in the titration period with glargine U300 vs insulin degludec, while no evaluation of severe hypoglycaemia was conducted as only one event was recorded during the trial. In addition, the dose of insulin degludec was lower than the dose of glargine U300 at the end of the trial by $0.11 \mathrm{U} / \mathrm{kg}$.

The primary objective of the Trial Comparing the Efficacy and Safety of Insulin Degludec and Insulin Glargine 300 Units/ml in Subjects with Type 2 Diabetes Mellitus Inadequately Treated with Basal Insulin and Oral Antidiabetic Drugs (CONCLUDE), a randomised head-tohead clinical trial, was to investigate the effect of insulin degludec $200 \mathrm{U} / \mathrm{ml}$ (degludec U200) and glargine U300 on hypoglycaemia in insulin-treated individuals with type 2 diabetes.

\section{Methods}

\section{Trial design}

Detailed methods of CONCLUDE have been described previously [22]. Briefly, this was a treat-to-target, randomised, open-label, active comparator-controlled trial that was conducted at 229 sites in 11 countries. The original 58 week trial duration comprised 52 weeks of active treatment with designation of the first 16 weeks as the titration period and the remaining 36 weeks as the maintenance period (hereafter referred to as the 'variable maintenance period'). In February 2018, a protocol amendment led to the extension of the trial, resulting in a total trial duration of up to 94 weeks with up to 88 weeks of active treatment, including a new maintenance period (hereafter referred to as the 'maintenance period') of 36 weeks. A detailed rationale for this amendment has been published previously [22] and the key reasons for the amendment are outlined in ESM Fig. 1. In brief, routine monitoring of blinded data showed an unusual pattern in the reporting of glycaemic variables and hypoglycaemic events. Specifically, the glycaemic data were inconsistent between centrallaboratory-measured variables $\left(\mathrm{HbA}_{1 \mathrm{c}}\right.$ and fasting plasma glucose [FPG]) and patient-reported fasting self-measured blood glucose (SMBG) values. Data available from SMBG monitoring indicated to the patient that the blood glucose levels were higher than they actually were, potentially increasing the risk of hypoglycaemia as a result of unnecessary insulin up-titration. At the time of the amendment, the number of patient-reported hypoglycaemic events confirmed by blood glucose was low while the number of pseudohypoglycaemic events (blood glucose $>3.9 \mathrm{mmol} / 1$ with symptoms) was high compared with the SWITCH 2 trial (comparing the effect of insulin degludec vs insulin glargine U100 on in individuals with type 2 diabetes) [10]. These observations, seen in general across the entire trial population, were related to the glycaemic data collection system (MyGlucoHealth blood glucose meter and electronic diary). Therefore, because of these safety concerns, the glycaemic data collection system was discontinued during the variable maintenance period. This system was replaced with an Abbott blood glucose meter and paper diary to be used for the remainder of the trial. To accommodate these changes, preserve the scientific integrity of the trial and ensure sufficient data collection for the confirmatory endpoints using the same glycaemic data collection system (Abbott blood glucose meter and paper diary), a new 36 week maintenance period was included in the trial. At the time of the amendment, recruitment had been finalised and all participants on treatment had completed the titration period. The duration of the variable maintenance period was dependent on each participant's individual randomisation date and/or approval of the amended protocol by health authorities and local ethics committees, if applicable. After implementation of the amended protocol, participants were asked to come in and initiate the maintenance period as soon as the resources were available at the trial site, irrespective of the next planned visit. Thus, all participants were not required to have all visits scheduled between weeks 16 and 52. The trial data remained blinded at the point of discovering the issue with the glycaemic data collection system and the implementation of the protocol amendment. No unplanned interim analysis of the trial data from the titration period was conducted. The primary endpoint (number of severe or blood-glucose-confirmed symptomatic hypoglycaemic events) at the completion of the maintenance period was evaluated utilising the same analysis duration and statistical methods as the original protocol. Changes were implemented to maintain participant safety and protect the scientific integrity of the trial.

CONCLUDE is registered with ClinicalTrials.gov no. NCT03078478. The trial was conducted in accordance with the Declaration of Helsinki and ICH Good Clinical Practice Guideline $[23,24]$. The protocol was approved by independent ethics committees or institutional review boards for each centre; written informed consent was obtained from each participant before any trial-related activities.

\section{Participants and treatments}

Eligible participants included adults aged $>18$ years with type 2 diabetes with $\mathrm{HbA}_{1 \mathrm{c}} \leq 80 \mathrm{mmol} / \mathrm{mol}(9.5 \%), \mathrm{BMI} \leq 45 \mathrm{~kg} / \mathrm{m}^{2}$ and treated with basal insulin (once or twice daily; NPH insulin, insulin detemir, glargine U100) with or without oral glucose-lowering drugs (OADs) at stable doses (any combination of metformin, dipeptidyl peptidase- 4 inhibitor, $\alpha$ glucosidase inhibitor, thiazolidinedione and sodium-glucose cotransporter 2 inhibitor) for at least 90 days. In addition, participants had to fulfil at least one risk criterion for 
hypoglycaemia [22]. The main exclusion criteria were treatment with bolus or premixed insulin or with sulfonylureas/ glinides within 90 days before the screening visit, severe renal impairment (eGFR $<30 \mathrm{ml} \mathrm{min}^{-1}[1.73 \mathrm{~m}]^{-2}$ ), or impaired liver function (alanine aminotransferase or aspartate aminotransferase $\geq 2.5$ times the upper limit of normal).

Consenting participants were randomised using a trialspecific, interactive-voice, web-response system. Participants were randomised 1:1 to receive degludec U200 (Novo Nordisk, Bagsvaerd, Denmark; the 100 U/ $\mathrm{ml}$ and $200 \mathrm{U} / \mathrm{ml}$ concentrations of degludec are bioequivalent and interchangeable [25-27]) or glargine U300 (Sanofi, Paris, France) administered once daily. Within each treatment arm, participants were randomised 1:1 to administer basal insulin either in the morning (from waking to breakfast) or in the evening (from main evening meal to bedtime). The same dosing time was maintained for each participant throughout the trial. When initiating degludec U200, the pre-trial daily basal insulin dose was reduced by $20 \%$, as per the protocol, irrespective of prior insulin type. Glargine U300 was initiated according to its label: unit-to-unit switch for participants on once-daily basal insulin; 20\% reduction for those on twice-daily NPH insulin (US patients) or any twice-daily basal insulin (European and Canadian patients). The insulin dose was titrated similarly for both insulins: once-weekly titration was based on the mean of three pre-breakfast SMBG measurements, with a fasting blood glucose target of $4.0-5.0 \mathrm{mmol} / 1$. The insulin dose was adjusted in multiples of $2 \mathrm{U}$ ranging from $-4 \mathrm{U}$ to $+8 \mathrm{U}$ depending on the mean pre-breakfast SMBG level [22]. The type and dose of pre-trial OADs remained unchanged throughout the trial unless safety reasons required a change.

\section{Endpoints}

The primary endpoint was the rate of overall symptomatic hypoglycaemic events (defined as severe [an event requiring third-party assistance [28]] or confirmed blood glucose $<3.1 \mathrm{mmol} / 1$ [with symptoms]) during the maintenance period. Secondary confirmatory hypoglycaemia endpoints included the rate of nocturnal symptomatic hypoglycaemic events (severe or blood-glucoseconfirmed with symptoms, occurring between 00:01 and $05: 59 \mathrm{~h}$ ) and the rate of severe hypoglycaemic events during the maintenance period. Overall symptomatic, nocturnal symptomatic and severe hypoglycaemic events were also assessed during the total treatment period (up to 88 weeks) as secondary endpoints. Other secondary endpoints included change from baseline to end of treatment in $\mathrm{HbA}_{1 \mathrm{c}}$ level and FPG level, basal insulin dose at the end of treatment, pre-breakfast SMBG level and body weight. The composite endpoints
$\mathrm{HbA}_{1 \mathrm{c}}<53 \mathrm{mmol} / \mathrm{mol}(7.0 \%)$ with no overall symptomatic hypoglycaemia and $\mathrm{HbA}_{1 \mathrm{c}}<53 \mathrm{mmol} / \mathrm{mol}(7.0 \%)$ with no nocturnal symptomatic hypoglycaemia were assessed during the maintenance period. The number of adverse events between the two treatment arms was also assessed during the trial period. An independent external event adjudication committee validated the following selected adverse events in a blinded manner: fatal events and severe hypoglycaemia.

\section{Statistical analysis}

The statistical analyses of the primary and secondary endpoints have been described previously [22]. Endpoints related to hypoglycaemia and safety endpoints were summarised using the safety analysis set; efficacy endpoints were summarised using the full analysis set. Statistical superiority testing of the primary and confirmatory secondary endpoints was performed following a hierarchical testing procedure to control the family-wise type I error rate in the strong sense and has been described previously [22]. The sample size was calculated to ensure at least $80 \%$ power for the primary endpoint analysis.

A negative binomial model with pre-trial OADs, region, sex and dosing time as fixed effects, age as covariate and logarithm of the exposure time as offset was used to estimate the rate ratio (RR) of hypoglycaemic events during the maintenance and total treatment periods. Participants with no on-treatment data during the maintenance period had values imputed for the maintenance period analyses based on participants discontinuing treatment during the maintenance period. Multiple imputations were performed using standard methods aligned with the analyses and planned to create 1000 complete datasets. The results were then combined using Rubin's methods [29]. The proportion of participants experiencing hypoglycaemic events was analysed post hoc using a logistic regression model. The model included treatment, pre-trial OADs, region, sex and dosing time as fixed effects and age as a covariate, and logarithm of the exposure time as offset. Change from baseline to end of treatment in $\mathrm{HbA}_{1 \mathrm{c}}$ levels, FPG levels, SMBG and body weight were analysed post hoc using mixed models for repeated measures (MMRM) with treatment, pre-trial OADs, region, sex and dosing time as fixed effects, and age and baseline $\mathrm{HbA}_{1 \mathrm{c}} / \mathrm{FPG}$ as covariates. Pre-specified sensitivity analyses were also conducted to test the primary and protocol-specified confirmatory secondary hypoglycaemia endpoints without imputed data as well as capping the number of hypoglycaemic events at three. Further post hoc sensitivity analyses controlling for variation across sites were conducted for $\mathrm{HbA}_{1 \mathrm{c}}$ and FPG. 


\section{Results}

\section{Participants}

Of the 2008 eligible participants screened, 1609 were randomised to receive either degludec $\mathrm{U} 200(n=805)$ or glargine U300 ( $n=804)$ (Fig. 1). A total of 1467 participants $(91.2 \%)$ completed the trial of whom $1409(87.6 \%)$ completed the trial on treatment. The proportion of participants withdrawing from the trial and discontinuing treatment prematurely was similar for both treatment groups. The protocol amendment did not have an apparent impact on participant retention rates (Fig. 1).

The characteristics of the participants at baseline were similar in the two treatment groups (Table 1) and did not differ between the randomised population and those entering the maintenance period (ESM Table 1). The mean age was 62.8 years, the mean duration of diabetes was 15.1 years, and the mean $\pm \mathrm{SD} \mathrm{HbA}_{1 \mathrm{c}}$ level was $59.2 \pm 10.5 \mathrm{mmol} / \mathrm{mol}$ $(7.6 \pm 1.0 \%)$. At screening, most participants were using glargine U100 (65.0\%) and were treated with metformin $(77.5 \%)$.

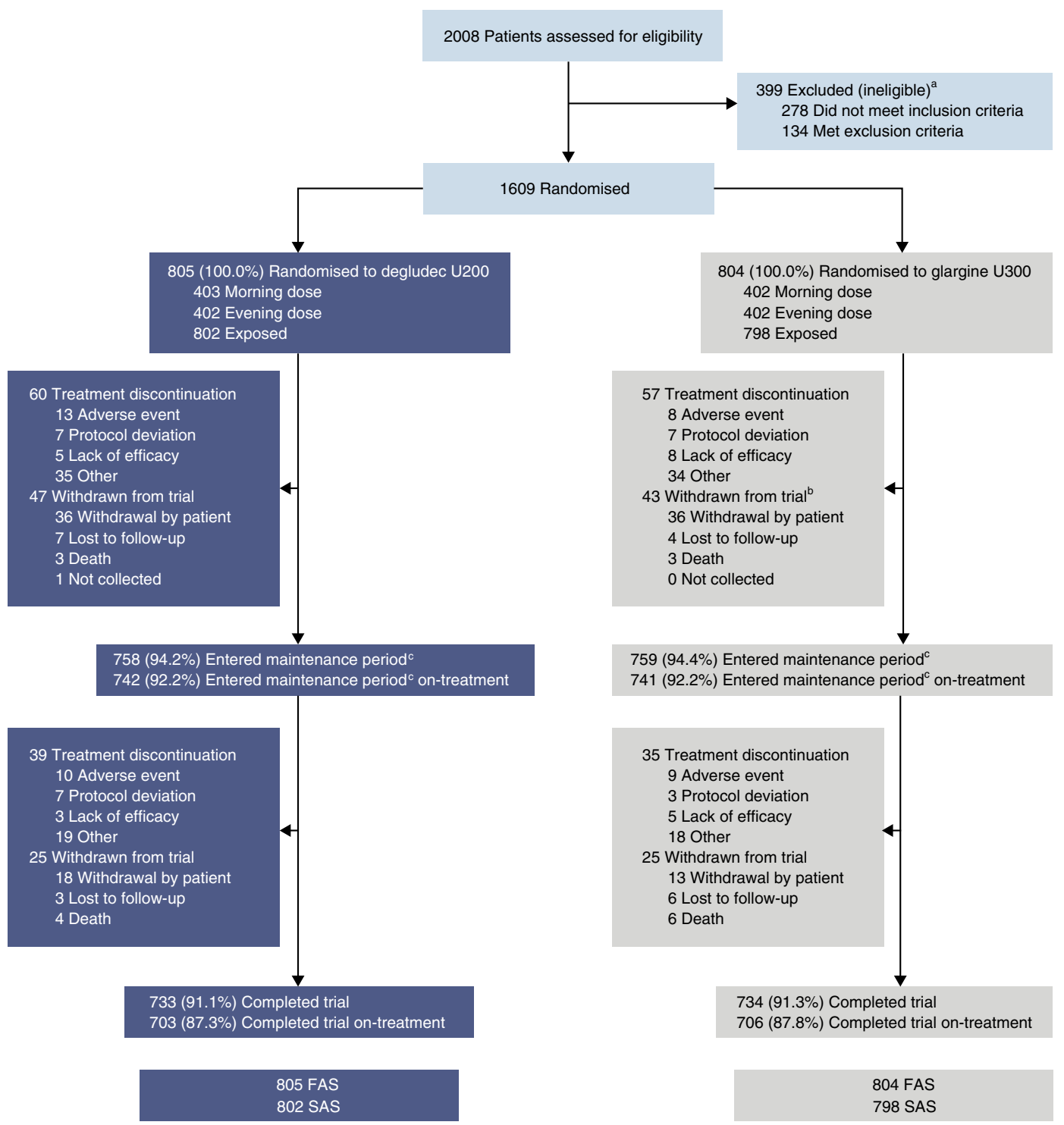

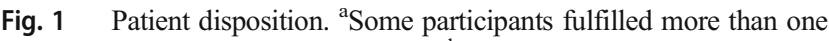
inclusion or exclusion criterion. ${ }^{\mathrm{b}}$ Two additional participants discontinued treatment before the protocol amendment and neither withdrew nor re-consented. ${ }^{\mathrm{c}} \mathrm{New}, 36$ week maintenance period $(52-$ 88 weeks). The number of participants who entered the maintenance period $=($ participants randomised $)-($ participants withdrawn from the trial). The number of participants who entered the maintenance period on-treatment $=($ participants exposed $)-($ participants who discontinued

treatment). The number of participants who completed trial $=$ (participants who entered the maintenance period) - (participants withdrawn from the trial). The number of participants who completed trial on-treatment $=$ (participants who entered the maintenance period on treatment $)-$ (participants who discontinued treatment). Exposed was defined as 'randomised and received treatment'. The number of participants that discontinued treatment includes the number that withdrew from the trial. FAS, full analysis set; SAS, safety analysis set 
Table $1 \quad$ Baseline characteristics of participants

\begin{tabular}{|c|c|c|c|}
\hline Characteristic & $\begin{array}{l}\text { Degludec U200 } \\
(N=805)\end{array}$ & $\begin{array}{l}\text { Glargine U300 } \\
(N=804)\end{array}$ & $p$ value \\
\hline Age, years & $62.9 \pm 10.0$ & $62.8 \pm 10.0$ & 0.8599 \\
\hline Men & $472(58.6)$ & $436(54.2)$ & 0.0785 \\
\hline Hispanic or Latino ethnicity & $84(10.4)$ & $100(12.4)$ & 0.2111 \\
\hline Race & & & 0.6978 \\
\hline White & $693(86.1)$ & $699(86.9)$ & \\
\hline Black or African-American & $78(9.7)$ & $65(8.1)$ & \\
\hline Asian & $25(3.1)$ & $29(3.6)$ & \\
\hline Other & $9(1.1)$ & $11(1.4)$ & \\
\hline Diabetes duration, years & $15.1 \pm 8.2$ & $15.0 \pm 8.4$ & 0.7676 \\
\hline Oral glucose-lowering treatment $\mathrm{t}^{\mathrm{a}}$ & $715(88.8)$ & $708(88.1)$ & 0.5835 \\
\hline Metformin & $622(77.3)$ & $625(77.7)$ & \\
\hline Dipeptidyl peptidase-4 inhibitor & $178(22.1)$ & $152(18.9)$ & \\
\hline SGLT-2 inhibitor & $150(18.6)$ & $153(19.0)$ & \\
\hline Combination of glucose-lowering treatments ${ }^{\mathrm{b}}$ & $41(5.1)$ & $44(5.5)$ & \\
\hline Thiazolidinedione & $37(4.6)$ & $25(3.1)$ & \\
\hline$\alpha$-Glucosidase inhibitors & $6(0.7)$ & $2(0.2)$ & \\
\hline Basal insulin & $803(99.8)^{\mathrm{c}}$ & $804(100.0)$ & 0.1014 \\
\hline Detemir & $171(21.2)$ & $139(17.3)$ & \\
\hline Glargine U100 & $505(62.7)$ & $541(67.3)$ & \\
\hline NPH insulin & $127(15.8)$ & $124(15.4)$ & \\
\hline Basal insulin dose, $\mathrm{U}$ & $42.7 \pm 29.5$ & $42.2 \pm 29.1$ & 0.7077 \\
\hline Body weight, kg & $91.6 \pm 18.1$ & $90.6 \pm 17.9$ & 0.2396 \\
\hline BMI, $\mathrm{kg} / \mathrm{m}^{2}$ & $31.7 \pm 5.3$ & $31.5 \pm 5.2$ & 0.5119 \\
\hline $\mathrm{HbA}_{1 \mathrm{c}}, \mathrm{mmol} / \mathrm{mol}$ & $59.0 \pm 10.8$ & $59.4 \pm 10.2$ & 0.5137 \\
\hline $\mathrm{HbA}_{1 \mathrm{c}}, \%$ & $7.6 \pm 1.0$ & $7.6 \pm 0.9$ & 0.5137 \\
\hline $\mathrm{FPG}, \mathrm{mmol} / \mathrm{l}$ & $7.9 \pm 2.6$ & $8.0 \pm 2.6$ & 0.6205 \\
\hline eGFR based on CKD-EPI ${ }^{\mathrm{d}}, \mathrm{ml} \mathrm{min}^{-1}[1.73 \mathrm{~m}]^{-2}$ & $78.8 \pm 21.2$ & $80.0 \pm 20.6$ & 0.2422 \\
\hline \multicolumn{4}{|c|}{ Participants fulfilling $\geq 1$ of the following hypoglycaemia risk inclusion criteria } \\
\hline$\geq 1$ severe hypoglycaemic event within the last year & $50(6.2)$ & $48(6.0)$ & \\
\hline Moderate chronic renal failure & $152(18.9)$ & $132(16.4)$ & \\
\hline Hypoglycaemia symptom unawareness & $166(20.6)$ & $141(17.5)$ & \\
\hline Exposed to insulin for $\geq 5$ years & $406(50.4)$ & $391(48.6)$ & \\
\hline Hypoglycaemic event within last 12 weeks & $466(57.9)$ & $479(59.6)$ & \\
\hline
\end{tabular}

Data are for the full analysis set and are shown as $n(\%)$ or mean \pm SD; percentage refers to the proportion of participants on degludec U200 or glargine $\mathrm{U} 300$ treatment. The $p$ value was determined by two-sided test of no difference

${ }^{a}$ One participant on sulfonylurea was randomised in error and discontinued treatment

${ }^{b}$ The combinations of glucose-lowering treatments includes allowed combinations, as per the inclusion criteria, only

${ }^{\mathrm{c}}$ One participant who was on premix NPH insulin and one patient who was insulin-naive were randomised in error

d Taken at screening

CKD-EPI, Chronic Kidney Disease Epidemiology Collaboration; SGLT-2, sodium-glucose cotransporter 2

\section{Hypoglycaemia endpoints}

Overall symptomatic hypoglycaemia For the primary endpoint, overall symptomatic hypoglycaemia, the rate was not significantly lower with degludec U200 compared with glargine U300 during the maintenance period (RR 0.88 [95\% CI 0.73, 1.06]) (Fig. 2). Because there was no significant difference between treatments for the primary endpoint, the confirmatory testing procedure for superiority was stopped. The pre-specified confirmatory secondary hypoglycaemia endpoints, nocturnal symptomatic and severe hypoglycaemia during the maintenance period, could not be controlled for the family-wise type I error and therefore were now considered exploratory. The sensitivity analyses conducted to test the 
Fig. 2 The rate of hypoglycaemia. Overall symptomatic hypoglycaemia was defined as severe hypoglycaemia (an event requiring third-party assistance as per the ADA definition [28]) or blood glucose $<3.1 \mathrm{mmol} / \mathrm{l}$ confirmed with symptoms. Nocturnal symptomatic hypoglycaemia was defined as severe hypoglycaemia or blood glucose $<3.1 \mathrm{mmol} / 1$ confirmed with symptoms, occurring between 00:01 and

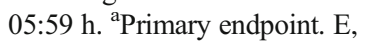
events; rate, events per 100 person-years of observation

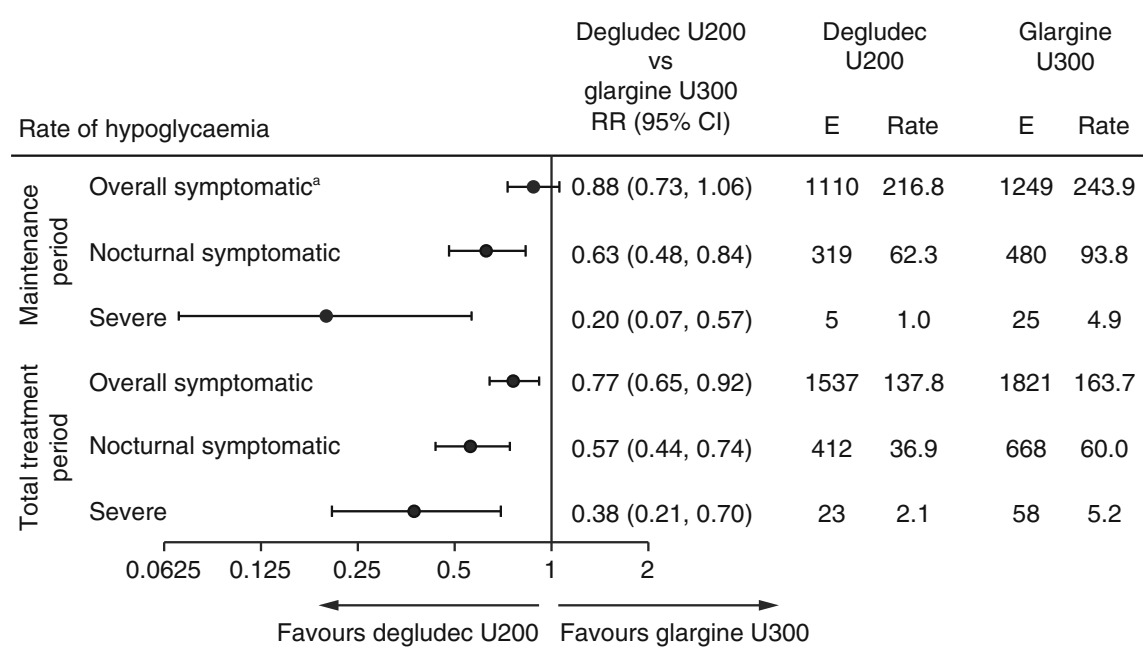

analyses conducted to test this endpoint without imputed data and capping the number of hypoglycaemic events at three showed similar results (ESM Table 2). The proportion of participants during the maintenance period experiencing nocturnal symptomatic hypoglycaemia was lower for those treated with degludec U200 (17.8\%) compared with glargine U300 (24.8\%): OR 0.65 (95\% CI 0.50, 0.83), post hoc analysis (Fig. 3). Similar results were observed during the total treatment period for the rate and the proportion of participants (post hoc) experiencing nocturnal symptomatic hypoglycaemia (Figs 2 and 3).

Severe hypoglycaemia The rate of severe hypoglycaemia was lower with degludec U200 compared with glargine U300 during the maintenance period (RR 0.20 [95\% CI 0.07 , 0.57]) (Fig. 2). The sensitivity analyses conducted to test this
Nocturnal symptomatic hypoglycaemia The rate of nocturnal symptomatic hypoglycaemia was lower with degludec U200 compared with glargine U300 during the maintenance period (RR 0.63 [95\% CI 0.48, 0.84]) (Fig. 2). The sensitivity primary endpoint without imputed data and capping the number of hypoglycaemic events at three showed similar The main analysis (ESM Table 2). lower for those treated with degludec U200 (40.6\%) compared with glargine U300 (46.3\%): OR 0.79 (95\% CI 0.64, 0.97), post hoc analysis (Fig. 3). During the total treatment period, the rate and the proportion of participants (post hoc) experiencing overall symptomatic hypoglycaemia was lower with degludec U200 vs glargine U300 (Figs 2 and 3).

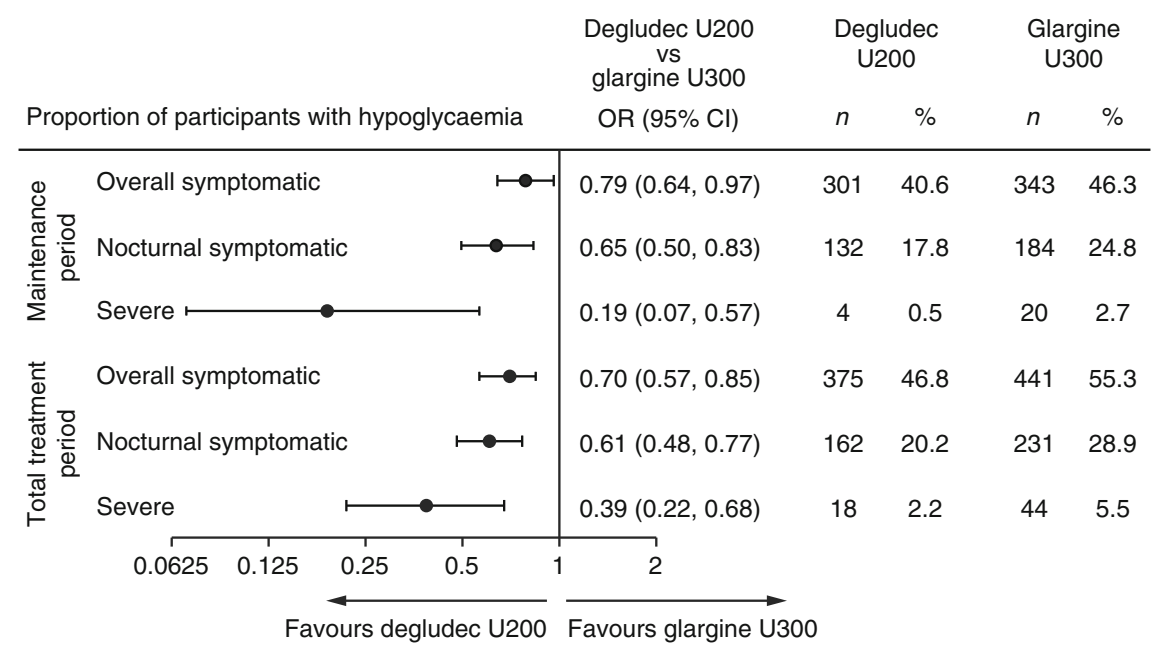

Fig. 3 The proportion of participants with hypoglycaemia (post hoc). Overall symptomatic hypoglycaemia was defined as severe hypoglycaemia (an event requiring third-party assistance as per the ADA definition [28]) or blood glucose $<3.1 \mathrm{mmol} / 1$ confirmed with symptoms. Nocturnal symptomatic hypoglycaemia was defined as severe hypoglycaemia or blood glucose $<3.1 \mathrm{mmol} / 1$ confirmed with symptoms, occurring between 00:01 and 05:59 h. \%, proportion of participants with events; $n$, number of participants experiencing events 


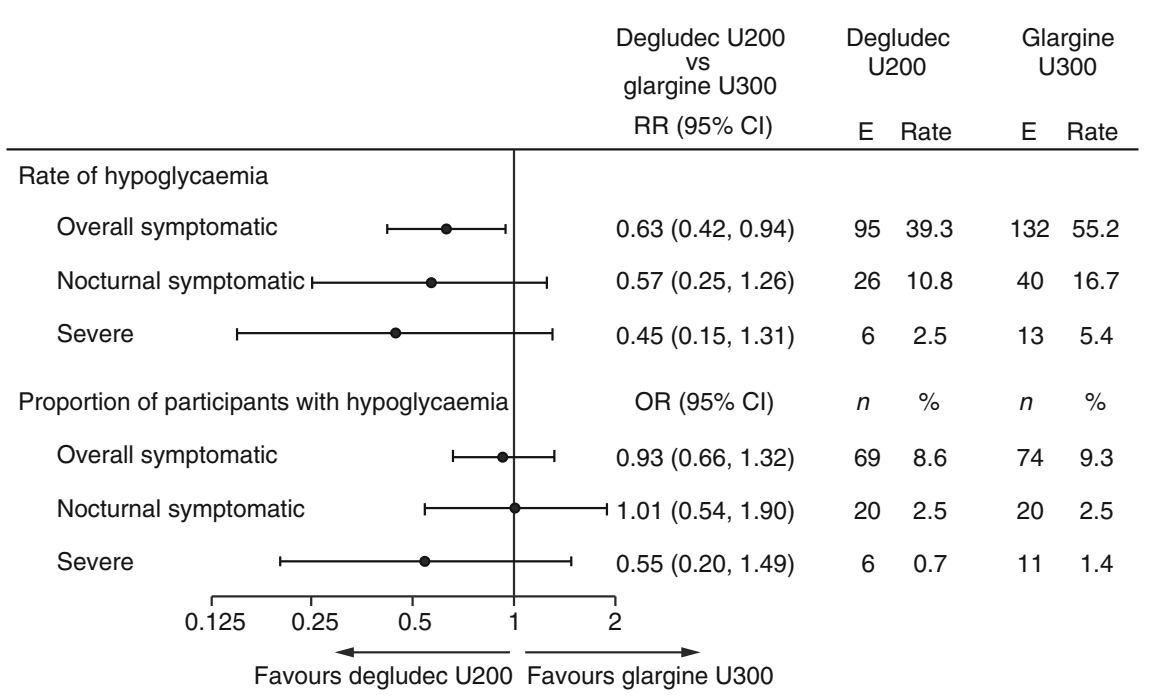

Fig. 4 Hypoglycaemia endpoints during the titration period. Overall symptomatic hypoglycaemia was defined as severe hypoglycaemia (an event requiring third-party assistance as per the ADA definition [28]) or blood glucose $<3.1 \mathrm{mmol} / \mathrm{l}$ confirmed with symptoms. Nocturnal symptomatic hypoglycaemia was defined as severe hypoglycaemia or blood glucose $<3.1 \mathrm{mmol} / 1$ confirmed with symptoms, occurring between 00:01 and 05:59 h. \%, proportion of participants with events; E, events; $n$, number of participants with events; rate, events per 100 person-years of observation

Hypoglycaemia during titration and variable maintenance periods The rates and the proportions of participants (post hoc) experiencing hypoglycaemia during the titration and variable maintenance periods are shown in Figs 4 and 5.

\section{Glycaemic control}

The observed mean $\mathrm{HbA}_{1 \mathrm{c}}$ values at the end of the titration period were $50.2 \mathrm{mmol} / \mathrm{mol}$ (6.8\%) with degludec U200 and (post hoc) experiencing severe hypoglycaemia (Figs 2 and 3).

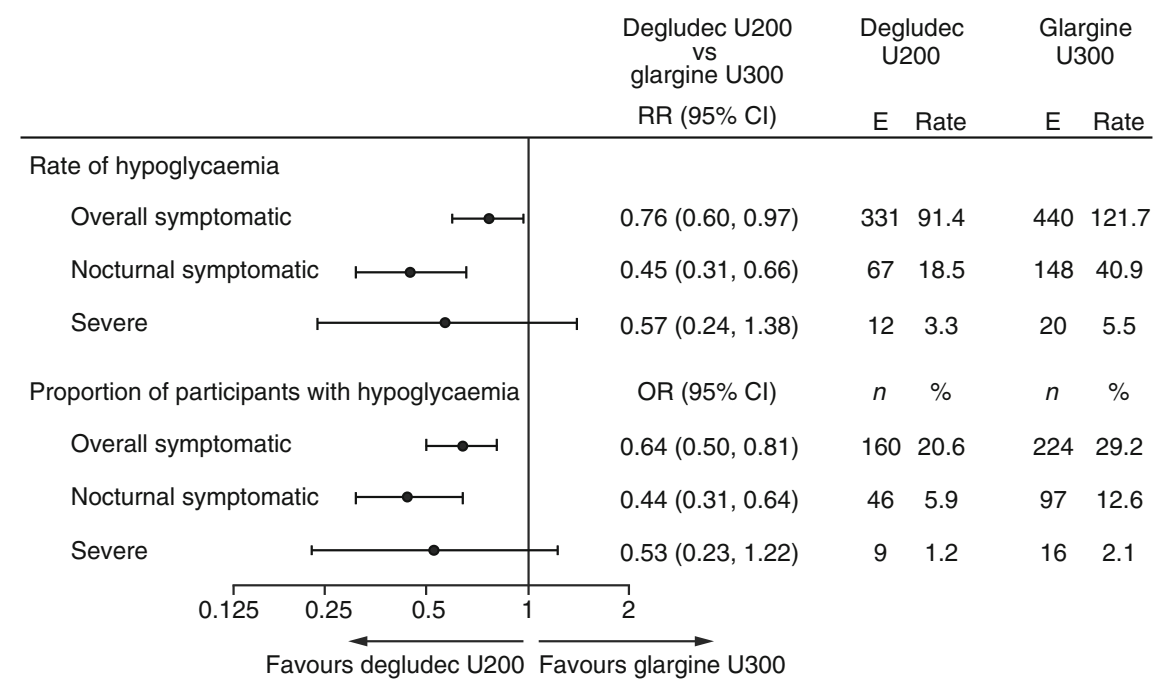

Fig. 5 Hypoglycaemia endpoints during the variable maintenance period. Overall symptomatic hypoglycaemia was defined as severe hypoglycaemia (an event requiring third-party assistance as per the ADA definition [28]) or blood glucose $<3.1 \mathrm{mmol} / 1$ confirmed with symptoms. Nocturnal symptomatic hypoglycaemia was defined as severe hypoglycaemia or blood glucose $<3.1 \mathrm{mmol} / 1$ confirmed with symptoms, occurring between 00:01 and 05:59 h. \%, proportion of participants with events; E, events; $n$, number of participants with events; rate, events per 100 person-years of observation 


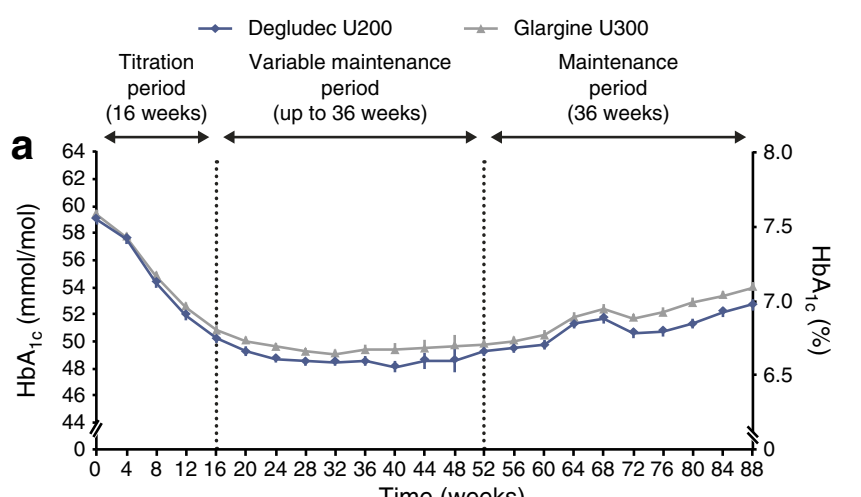

Degludec U200 ( $n$ ):

805786778776770765757716603506388267132739726723716702707704696692689 Glargine U300 (n):
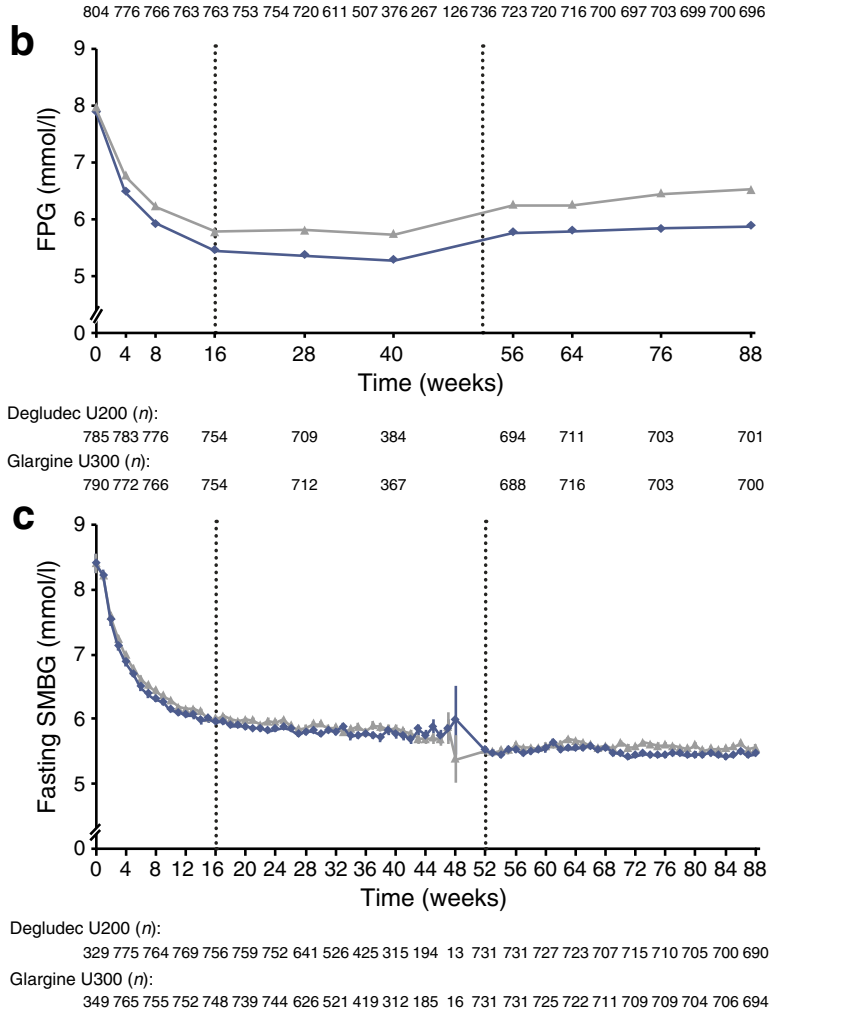

Fig. $6 \mathrm{HbA}_{1 \mathrm{c}}$, FPG and fasting $\mathrm{SMBG}$ over time. (a) $\mathrm{HbA}_{1 \mathrm{c}}$ over the study period. Estimated treatment difference from baseline to end of treatment for degludec U200 vs glargine U300 was $-1.07 \mathrm{mmol} / \mathrm{mol}$ $(95 \%$ CI $-1.94,-0.20)(-0.10 \%$ [95\% CI $-0.18,-0.02])$. (b) FPG over the study period. Estimated treatment difference from baseline to end of treatment for degludec U200 vs glargine U300 was $-0.62 \mathrm{mmol} / 1$ (95\% CI $-0.82,-0.43$ ). (c) SMBG over the study period. Estimated treatment difference from baseline to end of treatment for degludec U200 vs glargine U300 was $-0.18 \mathrm{mmol} / \mathrm{l}(95 \% \mathrm{CI}-0.37,0.01)$. Data are presented as mean \pm SEM, with the number of participants $(n)$ shown below each graph. Vertical dotted lines illustrate the end of the titration period (week 16) and the beginning of the maintenance period (week 52). According to the protocol, all participants were not required to complete all visits in the variable maintenance period and therefore the number of participants at each week decreased during this period

$50.9 \mathrm{mmol} / \mathrm{mol}(6.8 \%)$ with glargine U300. At the end of treatment, the observed mean $\mathrm{HbA}_{1 \mathrm{c}}$ was $52.8 \mathrm{mmol} / \mathrm{mol}$
(7.0\%) and $54.1 \mathrm{mmol} / \mathrm{mol}(7.1 \%)$ for degludec U200 and glargine U300, respectively. An analysis of $\mathrm{HbA}_{1 \mathrm{c}}$ demonstrated a reduction in the $\mathrm{HbA}_{1 \mathrm{c}}$ level from baseline to the end of treatment with degludec U200 compared with glargine U300: observed mean $-5.90 \mathrm{mmol} / \mathrm{mol}(-0.54 \%)$ vs $-5.04 \mathrm{mmol} / \mathrm{mol}(-0.46 \%)$; estimated treatment difference $-1.07 \mathrm{mmol} / \mathrm{mol}(95 \% \mathrm{CI}-1.94,-0.20)(-0.10 \%$ [95\% CI $-0.18,-0.02]$ ), post hoc analysis (Fig. 6a). At the end of treatment, the observed mean FPG was $5.9 \mathrm{mmol} / \mathrm{l}$ and $6.5 \mathrm{mmol} / \mathrm{l}$ for degludec U200 and glargine U300, respectively. There was also a reduction in FPG from baseline to the end of treatment with degludec U200 compared with glargine U300: observed mean $-1.97 \mathrm{mmol} / 1 \mathrm{vs}-1.43 \mathrm{mmol} / \mathrm{l}$; estimated treatment difference $-0.62 \mathrm{mmol} / 1$ ( $95 \%$ CI -0.82 , -0.43 ), post hoc analysis (Fig. 6b). Sensitivity analyses controlling for variation across study sites were conducted for $\mathrm{HbA}_{1 \mathrm{c}}$ and $\mathrm{FPG}$ and showed similar results to the main analysis (ESM Table 3). Over 88 weeks, pre-breakfast SMBG values were similar in the two treatment groups, decreasing during the titration period and then levelling off (estimated treatment difference $-0.18 \mathrm{mmol} / 1$ [95\% CI $-0.37,0.01]$, post hoc analysis) (Fig. 6c).

At the end of the maintenance period, $35.3 \%$ of participants treated with degludec U200 vs $30.0 \%$ of participants treated with glargine U300 achieved a composite endpoint of $\mathrm{HbA}_{1 \mathrm{c}}<53 \mathrm{mmol} / \mathrm{mol}(7.0 \%)$ with no overall symptomatic hypoglycaemia (OR 1.31 [95\% CI 1.04, 1.65], post hoc analysis). Similarly, $47.4 \%$ of participants treated with degludec U200 achieved an $\mathrm{HbA}_{1 \mathrm{c}}<53 \mathrm{mmol} / \mathrm{mol}$ (7.0\%) with no nocturnal symptomatic hypoglycaemia compared with $39.3 \%$ of participants treated with glargine U300 (OR 1.23 [95\% CI $0.99,1.54]$, post hoc analysis).

\section{Insulin dose}

The observed mean $\pm \mathrm{SD}$ baseline insulin dose for the degludec U200 and glargine U300 treatment arms was $42.7 \pm 29.5 \mathrm{U}$ and $42.2 \pm 29.1 \mathrm{U}$, respectively. At the start of treatment, the observed mean $\pm \mathrm{SD}$ basal insulin dose was $35.1 \pm 23.8 \mathrm{U}$ in the degludec U200 group and $42.4 \pm 29.2 \mathrm{U}$ in the glargine U300 group. At the end of treatment, the observed mean \pm SD dose was $66.6 \pm 48.5 \mathrm{U}$ for the degludec U200 group and 73.0 $\pm 48.5 \mathrm{U}$ for the glargine U300 group (Fig. 7).

\section{Adverse events and body weight}

The number of adverse events per 100 person-years of exposure (PYE) was 367.3 in the degludec U200 group and 365.4 per 100 PYE in the glargine U300 group; the corresponding rate of serious adverse events was 27.3 per 100 PYE vs 25.7 per 100 PYE, respectively (ESM Table 4). The most frequent adverse events $(\geq 5 \%)$ reported were nasopharyngitis, upper 
respiratory tract infection and diarrhoea. Serious adverse events attributed to hypoglycaemia during the trial were relatively infrequent ( 8 events [0.72 events/100 PYE] for degludec U200 and 21 events [1.89 events/100 PYE] for glargine U300). A total of seven participants (all on treatment) died in the degludec U200 group and nine (six on treatment) died in the glargine U300 group, of which no deaths were attributed to hypoglycaemic events or the trial products.

At the end of treatment, the observed change from baseline in body weight was higher in the degludec U200 group compared with the glargine U300 group (mean \pm SD: $2.9 \pm 5.2 \mathrm{~kg}$ vs $1.7 \pm$ $5.8 \mathrm{~kg})$, with an estimated treatment difference of $1.18 \mathrm{~kg}(95 \%$ CI $0.60,1.75$; post hoc analysis).

\section{Discussion}

In this open-label, randomised, treat-to-target trial in individuals with type 2 diabetes treated with insulin, administration of degludec U200 resulted in no significant difference in the rate of overall symptomatic hypoglycaemia but it did result in a nominally significantly lower rate of nocturnal symptomatic and severe hypoglycaemia during the maintenance period compared with glargine U300. In the total treatment period, the rate of hypoglycaemia was lower with degludec U200 for all three hypoglycaemia endpoints compared with glargine U300. Similarly, post hoc analyses showed that a lower proportion of participants experienced all three hypoglycaemia endpoints during the maintenance and total treatment periods. These hypoglycaemia results were achieved in the present trial without compromising glycaemic control.

Although the rate of overall symptomatic hypoglycaemia during the maintenance period (primary endpoint) was lower with degludec U200 compared with glargine U300, the difference did not reach statistical significance. Thus, it was not possible to rule out the possibility of no effect of degludec U200 compared with glargine U300. However, the $95 \%$ CI for the RR, from 0.73 to 1.06, indicates no clinically significant harm with degludec U200.

The BRIGHT trial was the first randomised clinical trial that compared degludec U100 with glargine U300 in insulin-naive individuals [21]. The results from BRIGHT demonstrated a comparable primary outcome of glycaemic control along with similar rates and proportions of participants experiencing hypoglycaemia (any-time and nocturnal) with glargine U300 vs degludec U100 in both the total treatment period ( 24 weeks) and the maintenance period (12 weeks). However, a lower rate and proportion of participants experienced any-time hypoglycaemia during the titration period (12 weeks) with glargine U300 than with degludec U100. The same results for the titration period were not observed in CONCLUDE. Because BRIGHT was undertaken in insulin-naive individuals without a history of severe hypoglycaemia or hypoglycaemia unawareness, there were not enough severe hypoglycaemic events to evaluate this outcome. The higher rates of severe hypoglycaemic events in the insulin-experienced population of CONCLUDE, who had at least one hypoglycaemia risk factor (including a history of severe hypoglycaemia), permitted an evaluation of these events. In terms of the trial design, CONCLUDE had longer durations of trial, titration period and maintenance period than BRIGHT. The
Fig. 7 Basal insulin dose over time. Data are presented as mean \pm SEM, with the number of participants $(n)$ shown below the graph. Vertical dotted lines illustrate the end of the titration period (week 16) and the beginning of the maintenance period (week 52). According to the protocol, all participants were not required to complete all visits in the variable maintenance period and therefore the number of participants at each week decreased during this period

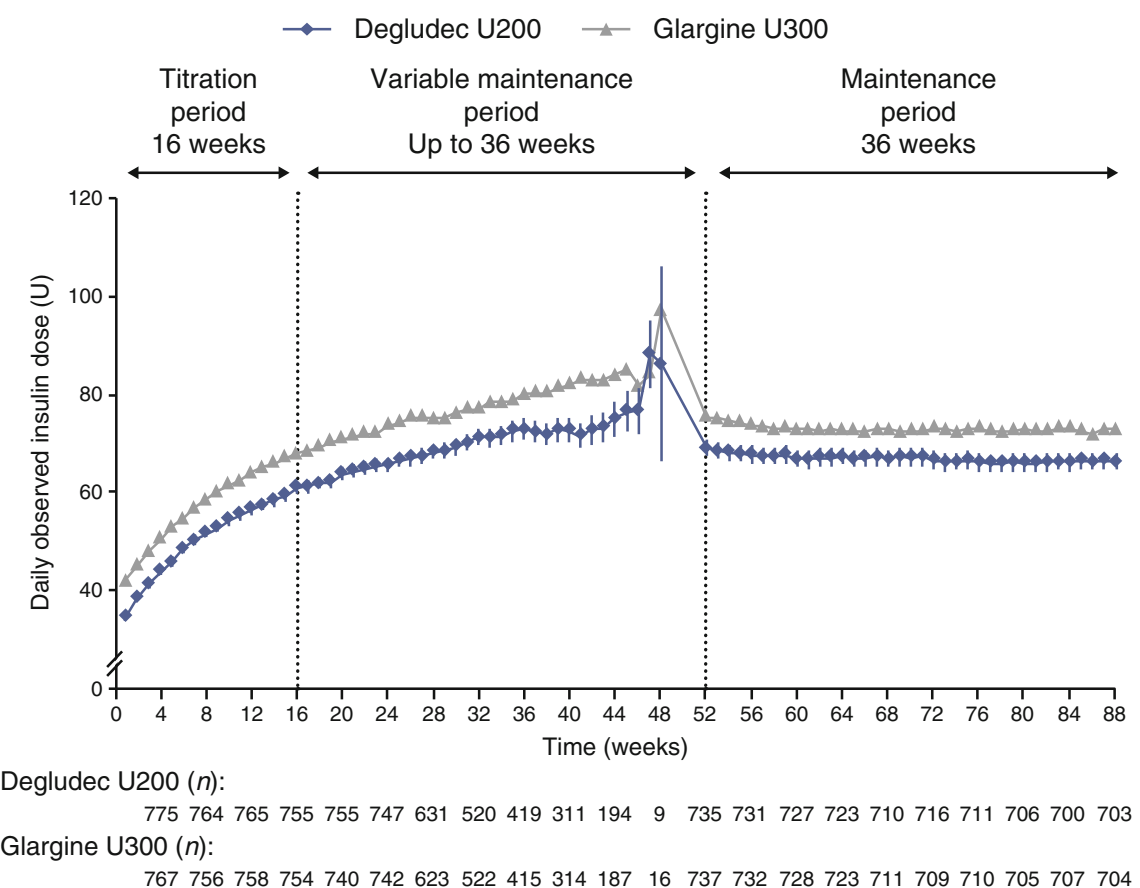

$767756758754740742623522415314187 \quad 16 \quad 737732728723711709710705707704$ 
two trials also had different primary endpoints: hypoglycaemia for CONCLUDE and $\mathrm{HbA}_{1 \mathrm{c}}$ for BRIGHT. Furthermore, the two trials used different definitions of hypoglycaemia with different blood glucose levels $(<3.1 \mathrm{mmol} / 1$ for CONCLUDE vs $\leq 3.9 \mathrm{mmol} / 1$ or $<3.0 \mathrm{mmol} / 1$ for BRIGHT) and had different titration algorithms. Target fasting SMBG in BRIGHT was 4.4-5.6 mmol/1, while in CONCLUDE the target was 4.0$5.0 \mathrm{mmol} / \mathrm{l}$. Finally, individuals treated with sulfonylureas were excluded from CONCLUDE while they were included in BRIGHT (65.7\% of participants at baseline).

Previous studies have demonstrated that glargine U300 has a weight benefit compared with glargine U100 [15-20]. During the CONCLUDE trial a greater increase in body weight was observed with degludec U200 compared with glargine U300.

This trial has several limitations. The requirement to amend the protocol and include an additional maintenance period added complexity to the trial. However, the change in protocol and trial design (i.e. the change in the glycaemic data collection system) was essential to protect the safety of the trial participants. Ultimately, we believe that these protocol revisions did not impact the scientific integrity of the trial because few participants $(n=25$ [1.6\%]) did not re-consent for the new maintenance period (between the day that the protocol amendment was implemented and each participant's individual day of initiating the new maintenance period; the remaining participants were withdrawn or lost to follow-up prior to the protocol amendment). However, we cannot exclude that unknown confounding factors could have been introduced that may have biased the perception of the investigators and participants, possibly affecting the conduct of the trial. In addition, it is important to note that although the MyGlucoHealth meter led to inaccurate blood glucose measurements, all participants used this system for the entire titration period thus the data reporting pattern was the same for both treatment groups. Moreover, the reporting of severe hypoglycaemia was not influenced by the inaccurate measurement, as these events were classified according to the ADA definition (requiring third-party assistance) and were externally adjudicated throughout the trial. Furthermore, degludec U200 and glargine U300 were compared in a controlled clinical trial setting, which limits the generalisability to routine clinical practice where individuals may have chronically higher $\mathrm{HbA}_{1 \mathrm{c}}$ levels despite basal insulin use, receive less support to prevent hypoglycaemia as well as use different titration targets and have issues regarding adherence. However, a recent literature review found that rates of hypoglycaemia overlap substantially in real-world settings and clinical trial settings [30]. Finally, data concerning the sociodemographic aspects (such as occupation, education level, etc.), which could potentially impact hypoglycaemia, were not collected during the trial.

Our trial has several strengths, including the large enrolment of insulin-treated individuals with a long duration of diabetes. The CONCLUDE population represents a more accurate reflection of patients seen in clinical practices than most published insulin randomised controlled trials where individuals with hypoglycaemia risk factors are typically excluded [9, 14-21]. Furthermore, the duration of the trial was relatively long, compared with most other clinical trials in this patient population, with a total treatment period of up to 88 weeks. This allowed for the assessment of glycaemic and hypoglycaemia outcomes over a longer time period than other trials.

In conclusion, the rate of overall symptomatic hypoglycaemia with degludec U200 was not significantly lower than with glargine U300 in the maintenance period. The rate of nocturnal symptomatic and severe hypoglycaemia were nominally significantly lower with degludec during the maintenance period compared with glargine U300.

Acknowledgements We thank the trial investigators, trial staff and trial participants for their participation, B. Anker Bak (employed by Biostatistics Insulin \& Devices, Novo Nordisk A/S, Denmark at the time of the trial) for statistical analysis and review and input to the manuscript, R. Kapoor and J. Gundgaard (Insulin \& Devices and HEOR Insulin, Novo Nordisk A/S, Denmark, respectively) for their review of and input to the manuscript, and F. Hemingway and C. Jones from Watermeadow Medical (sponsored by Novo Nordisk) for providing medical writing and editorial support. Some of the data from this trial were presented at the 55th EASD Annual Meeting in 2019.

Data availability The data generated during and/or analysed during the current trial are available from the corresponding author on reasonable request.

Funding This trial was sponsored and funded by Novo Nordisk (Bagsvaerd, Denmark). The trial sponsor was involved in the design of the trial, the collection and analysis of data, and writing the clinical report. Duality of interest APT has served on advisory panels for Eli Lilly and Co., Dexcom, Inc. and Voluntis, provided consultancy services for Novo Nordisk A/S and Sanofi US, and received research support from Merck \& Co., Inc., Novo Nordisk A/S, Sanofi US, Eli Lilly and Co., AstraZeneca, Janssen Pharmaceuticals, Inc. and Genentech, Inc. APT does not receive any direct or indirect payment for these services. DCK is a consultant for Abbott, Ascensia, EOFlow, Know, Lifecare, Merck, Novo Nordisk, Roche and Voluntis. KK has received honoraria from AstraZeneca, Boehringer Ingelheim, Eli Lilly, Janssen, Merck Sharp \& Dohme, Novartis, Novo Nordisk, Roche and Sanofi, and research support from AstraZeneca, Boehringer Ingelheim, Eli Lilly, Janssen, Merck Sharp \& Dohme, Novartis, Novo Nordisk, Roche and Sanofi, and also acknowledges support from the National Institute for Health Research Collaboration for Leadership in Applied Health Research and Care East Midlands (NIHR CLAHRC - EM) and the National Institute of Health Research (NIHR) Leicester Biomedical Research Centre. HSB reports speaking honoraria or research support from Abbott, Amgen, AstraZeneca, Boehringer Ingelheim, Eli Lilly, Janssen, Merck, Novo Nordisk and Sanofi. LAL has received research support from AstraZeneca, Boehringer Ingelheim, Eli Lilly, GSK, Janssen, Novo Nordisk and Sanofi, and has been on advisory panels and provided continuing medical education for AstraZeneca, Boehringer Ingelheim, Eli Lilly, Janssen, Merck, Novo Nordisk, Sanofi and Servier. MVH, LNT and SL are full-time employees of, and hold stock in, Novo Nordisk A/S. SH has served on speaker panels for Eli Lilly and Novo Nordisk, for which he has received remuneration. He has served on advisory panels or as a consultant for Zeeland, UNEEG Medical, Boehringer Ingelheim, Novo Nordisk, Eli Lilly, Sanofi Aventis and Takeda, for which his institution has received remuneration. TRP has received research support from Novo Nordisk and AstraZeneca (paid directly to the 
Medical University of Graz) and personal fees as a consultant from Adocia, Arecor, AstraZeneca, Eli Lilly, Novo Nordisk and Sanofi. TRP is also the Chief Scientific Officer of CBmed (Center for Biomarker Research in Medicine), a public-funded biomarker research company.

Contribution statement All authors confirm that they meet the International Committee of Medical Journal Editors uniform requirements for authorship. All authors made substantial contributions to the conception and design, acquisition of data or interpretation of data for this study. All authors had access to the final results and vouch for the fidelity of the trial to the protocol. All authors contributed to drafting/critically revising the article and approval of the final manuscript. APT is the guarantor of this work and, as such, had full access to all of the data in the trial and takes responsibility for the integrity and accuracy of the data, the final responsibility for the content of the manuscript and the decision to submit it for publication. Medical writing and editorial support, under the guidance of the authors, was provided by Watermeadow Medical, an Ashfield company, part of UDG Healthcare plc, funded by Novo Nordisk.

Open Access This article is licensed under a Creative Commons Attribution 4.0 International License, which permits use, sharing, adaptation, distribution and reproduction in any medium or format, as long as you give appropriate credit to the original author(s) and the source, provide a link to the Creative Commons licence, and indicate if changes were made. The images or other third party material in this article are included in the article's Creative Commons licence, unless indicated otherwise in a credit line to the material. If material is not included in the article's Creative Commons licence and your intended use is not permitted by statutory regulation or exceeds the permitted use, you will need to obtain permission directly from the copyright holder. To view a copy of this licence, visit http://creativecommons.org/licenses/by/4.0/.

\section{References}

1. Frier BM (2008) How hypoglycaemia can affect the life of a person with diabetes. Diabetes Metab Res Rev 24(2):87-92. https://doi. org/10.1002/dmrr.796

2. Leiter LA, Yale J-F, Chiasson J-L, Harris S, Kleinstiver P, Sauriol L (2005) Assessment of the impact of fear of hypoglycemic episodes on glycemic and hypoglycemia management. Can J Diabetes 29(3): 186-192

3. Becker RH, Dahmen R, Bergmann K, Lehmann A, Jax T, Heise T

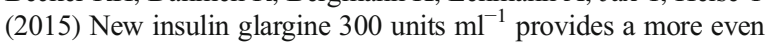
activity profile and prolonged glycemic control at steady state compared with insulin glargine 100 units $\mathrm{ml}^{-1}$. Diabetes Care 38(4):637-643. https://doi.org/10.2337/dc14-0006

4. Heise T, Hovelmann U, Nosek L, Hermanski L, Bottcher SG, Haahr H (2015) Comparison of the pharmacokinetic and pharmacodynamic profiles of insulin degludec and insulin glargine. Expert Opin Drug Metab Toxicol 11(8):1193-1201. https://doi.org/10. $1517 / 17425255.2015 .1058779$

5. Heise T, Nosek L, Bottcher SG, Hastrup H, Haahr H (2012) Ultralong-acting insulin degludec has a flat and stable glucose-lowering effect in type 2 diabetes. Diabetes Obes Metab 14(10):944-950. https://doi.org/10.1111/j.1463-1326.2012.01638.x

6. Heise T, Hermanski L, Nosek L, Feldman A, Rasmussen S, Haahr H (2012) Insulin degludec: four times lower pharmacodynamic variability than insulin glargine under steady-state conditions in type 1 diabetes. Diabetes Obes Metab 14(9):859-864. https://doi. org/10.1111/j.1463-1326.2012.01627.x
7. Heise T, Norskov M, Nosek L, Kaplan K, Famulla S, Haahr HL (2017) Insulin degludec: lower day-to-day and within-day variability in pharmacodynamic response compared with insulin glargine $300 \mathrm{U} / \mathrm{ml}$ in type 1 diabetes. Diabetes Obes Metab 19(7):10321039. https://doi.org/10.1111/dom.12938

8. Bailey TS, Pettus J, Roussel R et al (2018) Morning administration of $0.4 \mathrm{U} / \mathrm{kg} /$ day insulin glargine $300 \mathrm{U} / \mathrm{ml}$ provides less fluctuating 24-hour pharmacodynamics and more even pharmacokinetic profiles compared with insulin degludec $100 \mathrm{U} / \mathrm{mL}$ in type 1 diabetes. Diabetes Metab 44(1):15-21. https://doi.org/10.1016/j.diabet. 2017.10.001

9. Ratner RE, Gough SC, Mathieu C et al (2013) Hypoglycaemia risk with insulin degludec compared with insulin glargine in type 2 and type 1 diabetes: a pre-planned meta-analysis of phase 3 trials. Diabetes Obes Metab 15(2):175-184. https://doi.org/10.1111/ dom. 12032

10. Wysham C, Bhargava A, Chaykin L et al (2017) Effect of insulin degludec vs insulin glargine U100 on hypoglycemia in patients with type 2 diabetes: the SWITCH 2 randomized clinical trial. JAMA 318(1):45-56. https://doi.org/10.1001/jama.2017.7117

11. Gough SC, Bhargava A, Jain R, Mersebach H, Rasmussen S, Bergenstal RM (2013) Low-volume insulin degludec 200 units $/ \mathrm{ml}$ once daily improves glycemic control similarly to insulin glargine with a low risk of hypoglycemia in insulin-naive patients with type 2 diabetes: a 26-week, randomized, controlled, multinational, treatto-target trial: the BEGIN LOW VOLUME trial. Diabetes Care 36(9):2536-2542. https://doi.org/10.2337/dc12-2329

12. Marso SP, McGuire DK, Zinman B et al (2017) Efficacy and safety of degludec versus glargine in type 2 diabetes. N Engl J Med 377(8):723-732. https://doi.org/10.1056/NEJMoa1615692

13. Vora J, Christensen T, Rana A, Bain SC (2014) Insulin degludec versus insulin glargine in type 1 and type 2 diabetes mellitus: a meta-analysis of endpoints in phase 3a trials. Diabetes Ther 5(2): 435-446. https://doi.org/10.1007/s13300-014-0076-9

14. Riddle MC, Bolli GB, Ziemen M, Muehlen-Bartmer I, Bizet F, Home PD (2014) New insulin glargine 300 units/ml versus glargine 100 units $/ \mathrm{ml}$ in people with type 2 diabetes using basal and mealtime insulin: glucose control and hypoglycemia in a 6-month randomized controlled trial (EDITION 1). Diabetes Care 37(10): 2755-2762. https://doi.org/10.2337/dc14-0991

15. Riddle MC, Yki-Jarvinen H, Bolli GB et al (2015) One-year sustained glycaemic control and less hypoglycaemia with new insulin glargine $300 \mathrm{U} / \mathrm{ml}$ compared with $100 \mathrm{U} / \mathrm{ml}$ in people with type 2 diabetes using basal plus meal-time insulin: the EDITION 112 month randomized trial, including 6-month extension. Diabetes Obes Metab 17(9):835-842. https://doi.org/10.1111/dom.12472

16. Yki-Jarvinen H, Bergenstal R, Ziemen M et al (2014) New insulin glargine 300 units $/ \mathrm{mL}$ versus glargine 100 units $/ \mathrm{ml}$ in people with type 2 diabetes using oral agents and basal insulin: glucose control and hypoglycemia in a 6-month randomized controlled trial (EDITION 2). Diabetes Care 37(12):3235-3243. https://doi.org/ $10.2337 / \mathrm{dc} 14-0990$

17. Yki-Jarvinen H, Bergenstal RM, Bolli GB et al (2015) Glycaemic control and hypoglycaemia with new insulin glargine $300 \mathrm{U} / \mathrm{ml}$ versus insulin glargine $100 \mathrm{U} / \mathrm{ml}$ in people with type 2 diabetes using basal insulin and oral antihyperglycaemic drugs: the EDITION 2 randomized 12-month trial including 6-month extension. Diabetes Obes Metab 17(12):1142-1149. https://doi.org/10. 1111/dom.12532

18. Bolli GB, Riddle MC, Bergenstal RM et al (2015) New insulin glargine $300 \mathrm{U} / \mathrm{ml}$ compared with glargine $100 \mathrm{U} / \mathrm{ml}$ in insulinnaive people with type 2 diabetes on oral glucose-lowering drugs: a randomized controlled trial (EDITION 3). Diabetes Obes Metab 17(4):386-394. https://doi.org/10.1111/dom.12438

19. Ritzel R, Roussel R, Bolli GB et al (2015) Patient-level meta-analysis of the EDITION 1, 2 and 3 studies: glycaemic control and 
hypoglycaemia with new insulin glargine $300 \mathrm{U} / \mathrm{ml}$ versus glargine $100 \mathrm{U} / \mathrm{ml}$ in people with type 2 diabetes. Diabetes Obes Metab 17(9):859-867. https://doi.org/10.1111/dom.12485

20. Ritzel R, Roussel R, Giaccari A, Vora J, Brulle-Wohlhueter C, YkiJarvinen H (2018) Better glycaemic control and less hypoglycaemia with insulin glargine $300 \mathrm{U} / \mathrm{ml}$ vs glargine $100 \mathrm{U} / \mathrm{ml}$ : 1-year patient-level meta-analysis of the EDITION clinical studies in people with type 2 diabetes. Diabetes Obes Metab 20(3):541-548. https://doi.org/10.1111/dom.13105

21. Rosenstock J, Cheng A, Ritzel R et al (2018) More similarities than differences testing insulin glargine 300 units $/ \mathrm{mL}$ versus insulin degludec 100 units $/ \mathrm{mL}$ in insulin-naive type 2 diabetes: the randomized head-to-head BRIGHT trial. Diabetes Care 41(10): 2147-2154. https://doi.org/10.2337/dc18-0559

22. Philis-Tsimikas A, Stratton I, Norgard Troelsen L, Anker Bak B, Leiter LA (2019) Efficacy and safety of degludec compared to glargine 300 units $/ \mathrm{ml}$ in insulin-experienced patients with type 2 diabetes: trial protocol amendment (NCT03078478). J Diabetes Sci Technol 13(3):498-506. https://doi.org/10.1177/ 1932296819841585

23. World Medical Association (2013) Declaration of Helsinki: ethical principles for medical research involving human subjects. JAMA 310(20):2191-2194. https://doi.org/10.1001/jama.2013.281053

24. ICH (2001) ICH harmonised tripartite guideline: guideline for good clinical practice. J Postgrad Med 47(3):199-203
25. European Medicines Agency (2018) Summary of product characteristics. Available from https://www.ema.europa.eu/en/documents/ product-information/tresiba-epar-product-information_en.pdf, accessed 6 August 2019

26. Novo Nordisk A/S (2018) Highlights of prescribing information. Available from https://www.accessdata.fda.gov/drugsatfda_docs/ label/2018/203314s010lbl.pdf, accessed 6 August 2019

27. Korsatko S, Deller S, Koehler G et al (2013) A comparison of the steady-state pharmacokinetic and pharmacodynamic profiles of 100 and $200 \mathrm{U} / \mathrm{ml}$ formulations of ultra-long-acting insulin degludec. Clin Drug Investig 33(7):515-521. https://doi.org/10.1007/s40261013-0096-7

28. Seaquist ER, Anderson J, Childs B et al (2013) Hypoglycemia and diabetes: a report of a workgroup of the American Diabetes Association and the Endocrine Society. Diabetes Care 36(5): 1384-1395. https://doi.org/10.2337/dc12-2480

29. Little RJA, Rubin DB (2019) Statistical analysis with missing data, 2nd edn. Wiley, Hoboken

30. Elliott L, Fidler C, Ditchfield A, Stissing T (2016) Hypoglycemia event rates: a comparison between real-world data and randomized controlled trial populations in insulin-treated diabetes. Diabetes Ther 7(1):45-60. https://doi.org/10.1007/s13300-016-0157-z

Publisher's note Springer Nature remains neutral with regard to jurisdictional claims in published maps and institutional affiliations. 